\title{
Physical Exercise and its Impact on Psychology
}

\section{Trevor Archer*}

Department of Psychology, University of Gothenburg, Box 500, S-405 30 Gothenburg, Sweden

\begin{abstract}
Physical exercise impacts upon a plethora of psychological domains: cognition, emotion, development, motor, behavioral, clinical, lifespan and ageing and biological. In the present account, two aspects of exercise intervention are considered, cognitive and emotional with particular reference to conditions of abnormal behavior and brain disorder. Despite developmental disadvantages, it appears the regular and sustained exercise endowers lasting and resilient benefits for health and well-being.
\end{abstract}

Keywords: Exercise; Cognition; Performance; Emotion; Expression; Intervention

\section{Introduction}

The multi-dimensional impact of physical exercise upon psychology and behavioural science is linked to the manifest healthbeneficial expressions of physical exercise over individuals' life-cycles, whether normal or in ill-health, may be encapsulated within several domains of welfare: (i) exercise and academic and other cognitive performances, (ii) exercise and the developmental trajectory, (iii) exercise for the alleviation of affective disorders, and (iv) the epigenetic manifestations of physical exercise. Surprisingly, the effects of exercise may be determined relatively quickly: just eight weeks of pre-season training on body composition, physical fitness, anaerobic capacity, and isokinetic strength in collegiate taekwondo athletes in endurance gave improvements on all these parameters, as assessed by relative peak power and anaerobic capacity and angular velocity [1]. Psychological well-being, cognitive, emotional, motor, behavioral, clinical, recuperative, epigenetic and health domains all make considerable impact upon individuals' propensity for and compliance with regular exercise and physical activity and vice versa throughout the lifespan development [2-16]. Accordingly, physical exercise is viewed as a "scaffolding" construct that buttresses against illness and damage incurred under conditions, such as traumatic brain injury, clinical depression, developmental disorders or neurodegenerative diseases and aging, by shaping conditions for construction, damage control and reconstruction. It furnishes ongoing processes that are maintained across the lifespan through the applications and development of complementary, alternative biomarkers and neural networks for the attainment of selected functions and performances $[17,18]$. For example, [19], using data from the ageing population of the "Betula" study (Umeå, Sweden) have shown that (i) higher levels of physical activity score were related to greater connectivity in the posterior default-mode network, (ii) higher levels of physical activity score were related to larger gray matter volume of the posterior cingulate cortex, and (iii) higher levels of physical activity score were related to higher perfusion rate within the posterior cingulate cortex.

Studies derived from several cognitive domains underline the necessity of physical exercise, over a sedentary existence, in ensuring adequate functioning [20]. As a step contributing to improved cognitive performance, running induces neurogenesis in the dentate gyrus of the hippocampus [21,22]. In studies of $\mathrm{C}_{57} / \mathrm{B}_{16}$ mice, with or without access to running-wheels [23], observed a shift in new neuron networks and three-fold increments in neurogenesis in the dorsal, but not the ventral, dentate gyrus, whereas afferent-traced cell labeling doubled in number. Regional analysis indicated that running differentially affected specific inputs such that contextual, spatial and temporal information encoding by increasing adult hippocampal neurogenesis and by reorganization of new neuron circuitry was facilitated. In humans, hippocampal involvement is implicated in 'face-name' relational memory and visual episodic memory, which provided the most powerful relationships in the strong positive association between physical activity and episodic memory performance [24]. In studies of the detrimental effects of maternal deprivation in rodents for later inhibitory avoidance conditioning $[25,26]$ it has been shown that physical exercise prevented short-term and long-term deficits in aversive and recognition memory performance and attenuated oxidative stress damage induced by the the deprivation schedule [27]. In chronically-stressed mice, the coadministration of physical exercise with dietary supplements induced marked elevations in the number of double cortin-positive immature neurons in the dentate gyrus, the sectional area of the dentate gyrus and hippocampal CA1 region, in addition to an increased hippocampal brain-derived neurotropic factor messenger ribonucleic acid (mRNA) and serum vascular endothelial growth factor concentrations, thereby bestowing multiple benefits $[28,29]$. In older adults (mean age 81.6 years) with postural problems ('falling-down' disabilities), executive functioning has been shown to present a marker of resiliency in several noncognitive, affective domains predicting reductions in symptoms of depression and maintenance of instrumental activities of daily living; improved executive functioning was predicted by physical activity gait speed, physical exercise and improved maintenance of instrumental activities of daily living during follow-up [30]. There is evidence supporting the associations among physical activity/exercise, cognitive vitality, neural functioning, and the moderation of these associations by genetic factors whereby the former preserves and augments cognitive vitality and its related neural circuitry in older adults, with the preponderance of benefits seen for tasks/functions that are supported by the prefrontal cortex and the hippocampus [31].

Well-trained, regularly exercising individual exhibit lesser physiological and behavioral responses to stressful provocations

*Corresponding author: Archer T, Department of Psychology, University of Gothenburg, Box 500, S-405 30 Gothenburg, Sweden, Tel: +46 31 7864694; E-mail: trevor.archer@psy.gu.se

Received: January 02, 2016; Accepted: January 03, 2016; Published: January 10, 2016

Citation: Archer T (2016) Physical Exercise and its Impact on Psychology. Clin Exp Psychol 2: e104. doi:10.4172/2471-2701.1000e104

Copyright: (c) 2016 Archer T. This is an open-access article distributed under the terms of the Creative Commons Attribution License, which permits unrestricted use, distribution, and reproduction in any medium, provided the original author and source are credited. 
and more effective recovery from provocation than sedentary individuals [32-34]. In families identified by depressed parents, adolescent mental health problems were found to be commonplace; nevertheless, a large portion (20\%) of the adolescents expressed sustained good mental health with frequent exercise linked to parent positive expressed emotion, co-parent support, good-quality social relationships, and self-efficacy [35]. In a comparison of highly trained and sedentary young adult men, randomly assigned to either exercise on a treadmill at moderate intensity (60-70\% VO2max) for $30 \mathrm{~min}$, or to perform $30 \mathrm{~min}$ of a "placebo" exercise [36], obtained a significantly reduced cortisol response to the Montreal Imaging Stress Task (employing functional magnetic resonance imaging), which was inversely related to the previous exercise-induced $\alpha$-amylase and cortisol fluctuations in the treadmill-exercise group. They observed also higher bilateral hippocampus activity and lower prefrontal cortex activity in this group. Higher aerobic fitness, highly trained men, was associated with lower cortisol responses. The acute stress-buffering effect of exercise hinges upon those negative feedback mechanisms originating from the hippocampus and prefrontal cortex that direct the regulation of the hypothalamus-pituitary-adrenal (HPA) axis. In this context it was shown that treadmill running exercise yielded beneficial effects by ameliorating the anxiety effects and the dysregulation of HPA axis induced by early life exposure to di-(2-ethylhexyl)-phthalate in adolescent female rats [37]. The notion of Mental and Physical Training envisages dependent measures of aerobic fitness (as assessed by maximal rate of oxygen consumed) concurrent with reductions in the observed symptoms of depression and anxiety among troubled individuals [38,39]. Mental and Physical Training (two sessions / week over eight weeks) produced these improvements whereas similar improvements were not observed in a group of recently homeless women who did not participate in MAP Training [40]. Neuropsychiatric conditions present frequent co-morbid status with chronic systemic diseases exemplified by the high incidences of depression; anxiety and cognitive impairment complicate cardiovascular and metabolic disorders such as hypertension and diabetes mellitus [41]. Thus, the positive effects of active lifestyle that incorporated exercise and activity, upon nonpsychotic mental disorders in patients presenting chronic systemic diseases was described [42]. Finally, exercise combined with thiamine supplementation improved spatial memory performance and brainderived neurotropic factor and acetylcholine in the hippocampus of the stressed Wistar rats [43].

In conclusion, the alleviating effect of physical exercise over multiple symptom domains continues to furnish convincing evidence for this noninvasive, non-pharmacological intervention. For example, in a laboratory model of attention-deficit/hyperactive disorder presented by spontaneously-hypertensive rats, it was shown that 5,10 , or 21 days of access to a running wheel, but not 2 days, significantly reduced levels of the norepinephrine transporter in medial prefrontal cortex and demonstrated a robust habituation to a non-reinforced visual stimulus that was indistinguishable from normo-active rats whereas pretreatment with propranolol, $\beta$-adrenergic/noradrenergic receptor blocker, abolished the exercise-induced reduction in orienting behavior [44], thereby establishing the impact of exercise on both behavior and biomarker.

\section{References}

1. Seo MW, Jung HC, Song JK, Kim HB (2015) Effect of 8 weeks of pre-season training on body composition, physical fitness, anaerobic capacity, and isokinetic muscle strength in male and female collegiate taekwondo athletes. $J$ Exerc Rehabil 11: 101-107.

2. Archer $T$ (2011) Physical exercise alleviates debilities of normal aging and
Alzheimer's disease. Acta Neurol Scand 123: 221-238.

3. Archer $\mathrm{T}$ (2015) (a) physical exercise as an epigenetic factor determining behavioral outcomes. Clin Exp Psych 1: 1.

4. Archer T (2015) (b) Exercise alleviates autism spectrum disorders. Autism Open Access 5: 2.

5. Archer T (2014) Health benefits of physical exercise for children and adolescents. J Novel Psychother 4: 203.

6. Archer T (2012) (a) Influence of physical exercise on traumatic brain injury deficits: scaffolding effect. Neurotox Res 21: 418-434.

7. Archer T (2012) (b) Influence of physical exercise on traumatic brain injury deficits: scaffolding effect. Neurotox Res 21: 418-34.

8. Archer T (2015) (c) Exercise as therapy: health and well-being. 76-81

9. Archer T (2015) (d) Exercise influences in depressive disorders: symptoms biomarkers and telomeres. Clin Depress 1: 1.

10. Archer T, Garcia D (2014) Physical exercise influences academic performance and well-being in children and adolescents 1: 102.

11. Archer T, Garcia D (2015) (a) Exercise and dietary restrictions for promotion of neuro health benefits. Health 7: 136-152.

12. Archer T, Garcia D (2015) (b) Exercise intervention in traumatic brain injury Trauma \& Acute Care 1: 1-3

13. Archer T, Kostrzewa RM (2012) Physical exercise alleviates ADHD symptoms: regional deficits and development trajectory. Neurotox Res 21: 195-209.

14. Archer T, Kostrzewa RM (2015) Physical Exercise Alleviates Health Defects, Symptoms, and Biomarkers in Schizophrenia Spectrum Disorder. Neurotox Res 28: 268-280.

15. Archer T, Josefsson T, Lindwall M (2014) Effects of physical exercise on depressive symptoms and biomarkers in depression. CNS Neurol Disord Drug Targets 13: 1640-1653.

16. Garcia D, Archer T (2014) Positive affect and age as predictors of exercise compliance. Peer J 2: 694.

17. Burzynska AZ, Wong CN, Voss MW, Cooke GE, Gothe NP, et al. (2015) Physical Activity Is Linked to Greater Moment-To-Moment Variability in Spontaneous Brain Activity in Older Adults.

18. Voss MW, Weng TB, Burzynska AZ, Wong CN, Cooke GE, et al. (2015) Fitness but not physical activity, is related to functional integrity of brain networks associated with aging. Neuroimage.

19. Boraxbekk CJ, Salami A, Wåhlin A, Nyberg L (2015) Physical activity over a decade modifies age-related decline in perfusion, gray matter volume, and functional connectivity of the posterior default-mode network-A multimodal approach.

20. Loprinzi PD, Kane CJ (2015) Exercise and cognitive function: a randomized controlled trial examining acute exercise and free-living physical activity and sedentary effects. Mayo Clin Proc 90: 450-460.

21. Clemente Motta-Teixeira L, Takada SH, Machado-Nils AV, Nogueira MI, Xavier GF (2015) Spatial learning and neurogenesis: Effects of cessation of wheel running and survival of novel neurons by engagement in cognitive tasks.

22. Thomas AG, Dennis A, Rawlings NB, Stagg CJ, Matthews L, et al. (2015) Multi-modal characterization of rapid anterior hippocampal volume increase associated with aerobic exercise.

23. Vivar C, Peterson BD, van Praag H (2015) Running rewires the neuronal network of adult-born dentate granule cells.

24. Hayes SM, Alosco ML, Hayes JP, Cadden M, Peterson KM, et al. (2015) Physical Activity Is Positively Associated with Episodic Memory in Aging. J Int Neuropsychol Soc 21: 780-90.

25. Aguggia JP, Suárez MM, Rivarola MA (2013) Early maternal separation: neurobehavioral consequences in mother rats. Behav Brain Res 248: 25-31.

26. Benetti F, da Silveira CK, Rosa J, Izquierdo I (2015) Histamine acting on the basolateral amygdala reverts the impairment of aversive memory of rats submitted to neonatal maternal deprivation. Behav Brain Res 278: 83-89.

27. Neves BH, Menezes J, Souza MA, Mello-Carpes PB (2015) Physical exercise prevents short and long-term deficits on aversive and recognition memory and attenuates brain oxidative damage induced by maternal deprivation. Physio 
Behav 152: 99-105.

28. Hutton CP, Déry N, Rosa E, Lemon JA, Rollo CD, et al. (2015) Synergistic effects of diet and exercise on hippocampal function in chronically stressed mice. Neuroscience 308: 180-193

29. Inoue K, Hanaoka Y, Nishijima T, Okamoto M, Chang H, et al. (2015) Long-term mild exercise training enhances hippocampus-dependent memory in rats. Int $\mathrm{J}$ Sports Med 36: 280-285.

30. Best JR, Davis JC, Liu-Ambrose T (2015) Longitudinal Analysis of Physical Performance, Functional Status, Physical Activity, and Mood in Relation to Executive Function in Older Adults Who Fall. J Am Geriatr Soc 63: 1112-1120.

31. Prakash RS, Voss MW, Erickson KI, Kramer AF (2015) Physical activity and cognitive vitality. Annu Rev Psychol 66: 769-797.

32. Bauer ME, Muller GC, Correa BL, Vianna P, Turner JE, et al. (2013) Psychoneuroendocrine interventions aimed at attenuating immunosenescence. A review Biogerontology 14: 9-20.

33. Gerber M, Kellmann M, Elliot C, Hartmann T, Brand S, et al. (2014) Perceived fitness protects against stress-based mental health impairments among police officers who report good sleep. J Occup Health 55: 376-384.

34. Sciolino NR, Smith JM, Stranahan AM, Freeman KG, Edwards GL, et al. (2015) Galanin mediates features of neural and behavioral stress resilience afforded by exercise. 89 : $255-264$

35. Collishaw S, Hammerton G, Mahedy L, Sellers R, Owen MJ, et al. (2015) Mental health resilience in the adolescent offspring of parents with depression: a prospective longitudinal study.

36. Zschucke E, Renneberg B, Dimeo F, Wüstenberg T, Ströhle A (2015) The stress-buffering effect of acute exercise: Evidence for HPA axis negative feedback. Psycho-neuroendocrinology 51: 414-225.

37. Wang DC, Chen TJ, Lin ML, Jhong YC, Chen SC (2014) Exercise prevents the increased anxiety-like behavior in lactational di-(2-ethylhexyl) phthalateexposed female rats in late adolescence by improving the regulation of hypothalamus-pituitary-adrenal axis. Horm Behav 66: 674-684.

38. Lubans DR, Smith JJ, Morgan PJ, Beauchamp MR, Miller A, et al. (2015) Mediators of Psychological Well-being in Adolescent Boys. J Adolesc Health.

39. Rimes RR, de Souza Moura AM, Lamego MK, de Sa Filho AS, et al. (2015) Effects of Exercise on Physical and Mental Health, and Cognitive and Brain Functions in Schizophrenia: Clinical and Experimental Evidence. CNS Neurol Disord Drug Targets 14: 1244-1254.

40. Shors TJ, Olson RL, Bates ME, Selby EA, Alderman BL (2014) Mental and Physical (MAP) Training: a neurogenesis-inspired intervention that enhances health in humans. Neurobiol Learn Mem 115: 3-9.

41. RoyD, McCannU, HanD, RaoV (2015) Pathological Laughter and Crying and Psychiatric Comorbidity After Traumatic Brain Injury. J Neuropsychiatry Clin Neurosci 27: 299-303.

42. Teixeira RB, Marins JC, de Sá Junior AR, de Carvalho CJ, da Silva Moura TA et al. (2015) Improved cognitive, affective and anxiety measures in patients with chronic systemic disorders following structured physical activity 12: 445-454.

43. E Dief A, M Samy D, I Dowedar F (2015) Impact of exercise and vitamin b1 intake on hippocampal brain-derived neurotrophic factor and spatial memory performance in a rat model of stress. J Nutr Sci Vitaminol 61: 1-7.

44. Robinson AM, Buttolph T, Green JT, Bucci DJ (2015) Physical exercise affects attentional orienting behavior through noradrenergic mechanisms. Behav Neurosci 129: 361-367. 